\title{
Riesgos naturales y conservación de la arquitectura defensiva de tierra: aproximación a los daños causados por seísmos en la Alcazaba de Almería y en la muralla de La Hoya
}

Natural Risks and Conservation of Rammed-earth Defensive Architecture: Approach to the Damages Caused by Earthquakes in the Alcazaba of Almeria and the Wall of La Hoya

\author{
María Marcos Cobaleda ${ }^{a}$, María Lourdes Gutiérrez-Carrillo ${ }^{b}$, Emilio Molero Melgarejo ${ }^{c}$ \\ a Universidad de Málaga, Málaga, Spain, mmcobaleda@uma.es \\ b Universidad de Granada, Granada, Spain, mlgutier@ugr.es \\ ${ }^{c}$ Universidad de Granada, Granada, Spain, emiliomolero@ugr.es
}

\begin{abstract}
The aim of this work is to present the results obtained in the framework of the PREFORTI Project. The particular case analysed is the damages caused by earthquakes in the medieval fortifications of Almeria and the consequences on their conservation. Almeria is a zone of important seismic activity. This particularity has caused many problems to conserve its Islamic military architecture. Within this work, we include the most important earthquakes that have affected this heritage since the late fifteenth century and the constructions damaged -to a greater or lesser extent- by them, including their specific damages and an approach to their state of conservation. Due to its importance within the military constructions, we present the case study of the Alcazaba of Almeria and the wall of La Hoya, focusing on the second one. These paradigmatic constructions were widely affected by the earthquakes since the late fifteenth century. Beyond the analysis of the damages caused by the earthquakes and the different historical restorations to mitigate them, we include the emergency measures proposed in the framework of the PREFORTI Project for its better conservation against the different risks, as well as the microzonation mapping of the natural seismicity risk for the section of the wall of La Hoya in order to delve in the study of the vulnerability of this cultural asset against this natural risk, as a mechanism for its better preventive conservation.
\end{abstract}

Keywords: Almeria, medieval fortifications, earthquakes, PREFORTI Project.

\section{Introducción}

El objetivo de este trabajo es presentar los resultados obtenidos en el Proyecto PREFORTI en el caso concreto de los daños causados por seísmos en las fortificaciones medievales de Almería y cómo han afectado a su conservación. El territorio de Almería está situado en una zona de importante actividad sísmica, lo que ha ocasionado numerosos problemas para conservar su arquitectura militar islámica.
En una primera parte, recogemos los seísmos más importantes que han afectado a este patrimonio desde finales del siglo $\mathrm{XV}$, así como las construcciones concretas que se vieron dañadas en mayor o menor medida, incluyendo los deterioros provocados y una aproximación a su estado de conservación. Tras estos aspectos generales, en un segundo apartado, dado su lugar destacado en la arquitectura militar peninsular, pre- 
sentamos el estudio de caso del conjunto de la Alcazaba de Almería y la muralla de La Hoya, centrándonos en esta última. Además del análisis de los daños por terremotos, incluimos otras patologías que han afectado al bien y las diferentes restauraciones históricas realizadas para paliarlos, así como las medidas de emergencia propuestas en el marco del Proyecto PREFORTI para su mejor conservación ante los distintos riesgos que la atenazan, junto con la elaboración de la cartografía de microzonificación del riesgo natural de sismicidad para el tramo de la muralla de La Hoya, con el fin de ahondar en el estudio de la vulnerabilidad del bien ante este riesgo natural como mecanismo que redunde en su mejor conservación preventiva.

\section{De 1487 a 1804: seísmos históricos y su re- percusión en el patrimonio defensivo alme- riense de época islámica}

El hecho de que el territorio almeriense esté situado en una zona de intensa actividad sísmica ha provocado que, desde los últimos años de dominio islámico (la ciudad fue conquistada por los Reyes Católicos en 1489), su patrimonio se haya visto afectado por los distintos terremotos documentados desde 1487. Presentamos en este apartado un recorrido por estos seísmos ordenados cronológicamente, indicando en cada caso las construcciones concretas que se vieron afectadas. Dadas las limitaciones en cuanto al tipo de arquitectura analizada en el marco del Proyecto PREFORTI, restringida a la realizada en tierra, los ejemplos que presentamos responden únicamente a este tipo de fortificaciones islámicas.

Como hemos indicado, a pesar de estar documentada en las fuentes la existencia de terremotos en esta zona desde la Antigüedad ${ }^{1}$ (Espinar Moreno, 1994, pp. 124-135), el punto de partida de nuestro estudio es el acaecido en Almería en el año 1487, debido a su gran intensidad (grado IX). El hecho fue relatado por Münzer, quien describe cómo la ciudad quedó arruinada (Espinar Moreno, 1994, p. 132). El seísmo afectó especialmente a la Alcazaba, destruyendo el recinto palatino -aprovechado por los Reyes Católicos para construir en su lugar un castillo-, así como parte de las murallas (Espinosa Spínola, et al., 2006, pp. 46-48). Este sismo afectó igualmente a las estructuras de la muralla de La Hoya (Marcos Cobaleda, Gutiérrez-Carrillo, en revisión), así como a la mayor parte de las murallas de la ciudad.

El siguiente seísmo con efectos en la arquitectura defensiva de tierra fue el que tuvo lugar el 9 de noviembre de 1518, con epicentro en Vera, alcanzando una intensidad VIII-IX (Silva, Rodríguez Pascua, 2014, p. 80), viéndose especialmente afectadas las fortificaciones islámicas de Vera, Garrucha y Mojácar. En el caso de Vera, la localidad quedó totalmente destruida, incluida su fortaleza (hasta los cimientos) y sus murallas (Martínez Solares, 2001, pp. 15, 660), viéndose obligada la población a abandonar el emplazamiento y trasladar la ciudad a su actual ubicación (Silva, Rodríguez Pascua, p. 161). Tras el seísmo, la fortaleza fue profundamente reformada con mampostería y ladrillo, sufriendo posteriormente los efectos de la erosión natural y el abandono (IAPH). En Mojácar, se produjo el hundimiento de la fortaleza y la caída de prácticamente todas sus torres y partes de la muralla (Silva, Rodríguez Pascua, 2014, p. 162), siendo reformadas a lo largo del siglo XVI (en 1552 y 1559). A causa de la erosión natural, ha perdido su muralla norte y se encuentra lleno de escombros y vegetación (Marcos Cobaleda, Gutiérrez Carrillo, 2019). En Garrucha, los efectos se sintieron con menor intensidad (grado VI-VII), aunque se derrumbó por completo la Torre de la Garrocha, realizada en tierra (Silva, Rodríguez Pascua, 2014, p. 162), construyéndose posteriormente en su lugar el Castillo de las Escobetas (Espinosa Spínola, et al., 2006, p. 311).

Especial mención merece el terremoto del 22 de septiembre de 1522, por su gran intensidad (grado IX), con una magnitud de 6,5 y epicentro en Alhama de Almería (Silva, Rodríguez Pascua, 2014, p. 163), cuyos efectos se sintieron en gran parte de la provincia, causando numerosas víctimas (Espinar Moreno, 1994, p. 154) y viéndose afectadas las fortificaciones de tierra de Almería, Alboloduy, Fiñana, Terque y Tabernas. Los efectos fueron tales que las localidades quedaron prácticamente arrasadas, llegando incluso a provocar daños en lugares distantes, como la Alhambra y la Catedral de Granada (Silva, Rodrí- 
guez Pascua, 2014, p. 163). En Almería, las defensas de la ciudad quedaron muy dañadas, siendo los adarves de la muralla urbana prácticamente destruidos (Marcos Cobaleda, Gutiérrez Carri1lo, 2019). En el caso de la muralla de La Hoya, su estabilidad estructural se vio muy afectada, problema que se agravó con el seísmo que tuvo lugar unos años más tarde, el 13 de abril de 1529, y que también afectó a la Alcazaba. Ésta, al igual que la cerca urbana, sufrió una gran devastación, primero con el terremoto de 1522 y posteriormente con los seísmos de 1526 y 1529. Entre los efectos del primero, podemos contar la destrucción del Muro de la Vela, que separaba el primer y el segundo recintos de la Alcazaba. Tras este terremoto, en diciembre de 1522, Carlos V mandó reparar los destrozos de la fortaleza, destinando la suma de 200.000 maravedíes al año para las reparaciones, durante los siguientes diez años, lo que da una idea aproximada del alcance de los daños (Espinar Moreno, 1994, pp. 157-158). Nuevos seísmos afectaron a la fortaleza en 1533 y 1549, perdiéndose el remate medieval de la Torre del Espolón (Suárez Márquez, 2009, p. 245). En lo que respecta a Alboloduy, el terremoto de 1522 arruinó el Castillo de El Hizam (López Guzmán, 2002, p. 423), quedando en estado de abandono. Graves daños sufrió también la Alcazaba de Fiñana, entre ellos, el derribo de la Torre del Homenaje y parte del adarve de la muralla. Desde este momento, a pesar de experimentar varias reparaciones, la fortaleza quedó en mal estado. Con su abandono tras la expulsión de los moriscos, las casas comenzaron a adosarse a su muralla, provocando daños en el primitivo recinto (Marcos Cobaleda, Gutiérrez Carrillo, 2019). En relación a Terque, el Castillo de Marchena fue destruido por los efectos del terremoto de 1522. A partir de este momento, el recinto se vio afectado por la erosión natural y el expolio de materiales (IAPH). Por último, en Tabernas, el seísmo destruyó la Alcazaba, que posteriormente quedó inutilizada por los conflictos con los moriscos, hasta que se abandonó en el siglo XVII (López Guzmán, 2002, p. 590). En época contemporánea, el recinto sufrió una restauración poco afortunada, que supuso pérdidas de los materiales medieva- les y desvirtuó su aspecto original (Marcos Cobaleda, Gutiérrez Carrillo, 2019).

Otro sismo que tuvo lugar en tierras almerienses en el siglo XVI, aunque apenas encontramos referencias, es el de 1563. Su epicentro debió localizarse en las proximidades de la actual localidad de Albox, puesto que la única noticia sobre él es la proporcionada por Alcocer Martínez en 1941, quien menciona que el Castillico de Albox sufrió graves daños a causa del mencionado terremoto, no siendo reparado posteriormente (Alcocer Martínez, 1941, p. 71).

Ya en el siglo XVII, tenemos también noticia de los temblores en la ciudad de Almería a finales de diciembre de 1658, alcanzando una intensidad de grado VIII (Silva, Rodríguez Pascua, 2014 , p. 80). Estos provocaron nuevos daños en la Alcazaba y la muralla urbana de la ciudad (sobre todo en su zona oriental), con el derribo de varios torreones y lienzos de muralla (Espinar Moreno, 1994, p. 163). Sus efectos se relatan en un documento del 2 de enero de 1659 , que narra lo sucedido entre el $30 \mathrm{y}$ el 31 de diciembre del año anterior: "[...] Serían las una cuando la tierra giraba y arrastraba torres hacia el mar, y creímos sepultarnos en las olas, por lo cual huimos hacia la Alcazaba; pero esta se caía, rodaban murallas y su suelo se llenaba de grietas, sonando bloques en lo hondo" (Espinar Moreno, 1994, p. 163).

El 1 de noviembre de 1755 tuvo lugar el conocido como "Terremoto de Lisboa". Este fue el de mayores dimensiones documentado que haya afectado a la Península Ibérica, con epicentro situado al suroeste del Cabo de San Vicente, en el Océano Atlántico. Alcanzó una magnitud de 8,5 y una intensidad de grado XI-XII (Silva, Rodríguez Pascua, 2014, p. 169). Sus efectos se hicieron eco en los territorios de Almería, causando el derribo de parte de la muralla del tercer recinto de la Alcazaba (Torres López, 1992, p. 29). Asimismo, según narra el corregidor de Ugíjar, Juan Tamarie y Vargas, en una misiva fechada el 15 de diciembre de 1755, el municipio de Canjáyar se vio afectado por el seísmo, principalmente en lo que se refiere a su iglesia parroquial (Martínez Solares, 2001, p. 641), aunque muy probablemente también sufriera daños la fortale- 
za de El Castillejo, en la misma localidad. Su estado actual de conservación es muy deficiente, ya que en el siglo XIX prácticamente terminó de destruirse para construir una ermita (IAPH). Por el contrario, gracias a una carta escrita el 6 de diciembre de 1755 por Don Lope de Medieta, Obispo de Almería y dirigida al Gobernador, tenemos constancia de las localidades que no sufrieron daños ocasionados por el terremoto de Lisboa (Martínez Solares, 2001, p. 140). De todas ellas, en las que se ha documentado la existencia de construcciones militares de tierra -en las que, debido al carácter de la misiva, se sobreentiende que no hubo daños tras este gran seísmo- son las siguientes: Alhabia (Castillo Pago de los Nietos), Felix (Castillo de Felix), Gádor (El Castillejo), Gérgal (el Castellón); Níjar (Castillo de Huebro, Castillo del Peñón de Inox y Torre de los Alumbres), Rioja (Torre de las Doblas) y Santa Fe de Mondújar (Torre de Santa $\mathrm{Fe})$.

Tras el terremoto de Lisboa, otros sismos tuvieron lugar en la segunda mitad del siglo XVIII, aunque con una intensidad mucho menor. El 8 de enero de 1756, tuvo lugar un terremoto en Huércal-Overa, aunque no hay constancia de que éste causase daños en sus fortalezas de tierra: el Castillo de Urcal, el Castillo de Huércal-Overa y el Castillo de Santa Bárbara (García Asensio, 1908, pp. 445-446). Otros seísmos posteriores causaron algunos daños en la Alcazaba de Almería, como el de abril de 1768, que ocasionó graves desperfectos en las estructuras defensivas, o el del 9 de octubre de 1790 (Torres López, 1992, p. 30).

Ya en el siglo XIX, el 25 de agosto de 1804, tuvo lugar un terremoto con epicentro en Dalías, con una magnitud de 6,4, que alcanzó una intensidad de grado VIII-IX (Silva, Rodríguez Pascua, 2014, p. 171). Se desconoce si las fortalezas de tierra de la región (los Castillos Cerro Moriscas, de Dalías y del Cerroncillo, o la Torre de Aljízar) se vieron afectadas por el seísmo (Martínez Solares, 2001, p. 15), aunque sí está documentado el colapso total o parcial de las torres y casas de la localidad (Silva, Rodríguez Pascua, 2014, p. 171). En el caso del Castillo de Dalías, parece que sí se vería afectado, puesto que a inicios del siglo XIX se levantaron en el empla- zamiento algunas construcciones y se adaptó el terreno a eras para trillar, por lo que seguramente se encontrara en ruinas (López Guzmán, 2002, p. 505). Los daños de este terremoto afectaron a construcciones más lejanas, como la Torre de Roquetas, en Roquetas de Mar, que desde 1593 estaba embutida en la fábrica del Castillo de Santa Ana (Cara Barrionuevo, Rodríguez López, 1992, p. 147), y que quedó muy afectada tras este sismo (hasta la destrucción total del Castillo con otro terremoto a inicios del siglo $\mathrm{XX}$ ) (IAPH). Asimismo, el terremoto de 1804 afectó a las construcciones de la Alcazaba de Almería. Entre sus efectos, se encuentra la destrucción en este momento de la Torre de Poniente (Torres López, 1992, p. 30).

\section{Caso de estudio: la Alcazaba de Almería y la muralla de La Hoya}

Según hemos analizado, la Alcazaba de Almería y la muralla de La Hoya se vieron afectadas por gran parte de los seísmos acaecidos en el territorio de Almería desde 1487 a 1804. En el caso de la Alcazaba, la existencia de mayor documentación sobre esta construcción, recoge los daños causados por los terremotos de 1487, 1522, $1526,1529,1533,1549,1658$, el terremoto de Lisboa de 1755, y los sismos de 1768, 1790 y 1804 , recogidos todos ellos en el apartado anterior. Para el caso específico de la muralla de La Hoya en el que nos centramos en este apartado, los daños causados por los sismos documentados se limitan a los terremotos de 1487, 1522 y 1529 , afectando todos ellos a su estabilidad estructural. En relación a este riesgo natural, en el proyecto PREFORTI se ha elaborado la cartografía de microzonificación del riesgo de sismicidad a partir de la consideración de distintos rangos de la aceleración sísmica de cálculo para el sector de la muralla de La Hoya. Su geolocalización precisa ha permitido la puesta en relación con las variables territoriales. Para esta labor han sido seleccionadas coberturas existentes como bases de referencia vía WMS (Web Map Service) del Open Geospatial Consortium, posibilitando la confección de la cartografía de riesgo sísmico mediante diversos procesos de análisis espacial: interpolación, densidad kernel, extrapolación, intersección y disolución. De manera ge- 
nérica, ha sido de utilidad el empleo de coberturas primarias provenientes de diversas fuentes de información, ente ellas, el Instituto Geológico y Minero de España (IGME) y, en particular, del GEODE - Cartografía geológica digital continua, además de haberse obtenido las cartografías secundarias de microzonificación por elaboración propia. Este material vincula el bien con su contexto territorial, recogiendo la intensidad del factor de peligrosidad natural. En este caso, prácti- camente la totalidad del tramo se localiza en una zona donde la aceleración sísmica oscila entre $0,24 \mathrm{~g}$ y $0,38 \mathrm{~g}$, lo que se traduce en una alta intensidad del factor de peligrosidad, que, sumado a las características del propio bien y por tanto a su vulnerabilidad, provoca un alto índice de riesgo sísmico, cuestión ésta de gran importancia y a tener en cuenta en el diseño de futuras medidas para su conservación preventiva (Fig. 1).

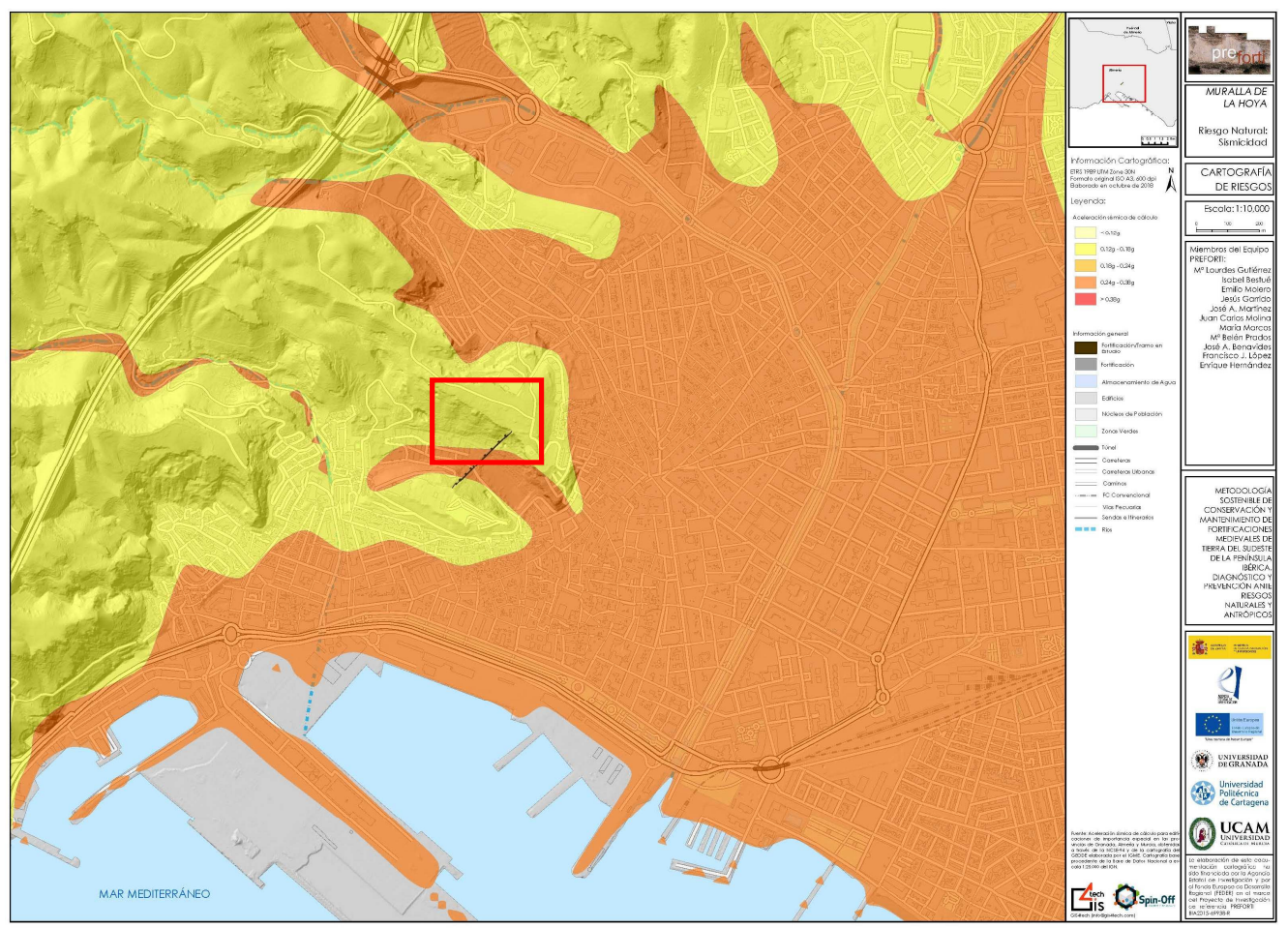

Fig. 1. Cartografía de microzonificación del riesgo natural de sismicidad para el tramo de la muralla de La Hoya, Almería (Proyecto PREFORTI, octubre 2018).

Junto a los daños causados por los seísmos, otros riesgos naturales y antrópicos han afectado a este lienzo a lo largo de los siglos, destacando los provenidos por la acción del agua, que ha provocado manchas de humedad, escorrentías y/o filtraciones facilitando a su vez notables abombamientos; los de origen estructural, que han favorecido la aparición de grietas y fisuras; o aquellos derivados de fenómenos erosivos por la labor del viento que ha causado pérdidas de materiales (Marcos Cobaleda, Gutiérrez Carrillo, en revisión). Para paliar estos y otros daños, a lo largo de la historia se han realizado varias intervenciones de conservación y restauración en este lienzo de muralla, que detallamos a continuación. Aunque la muralla de La Hoya se viene reparando desde finales del siglo XV y, sobre todo, desde el siglo XVI, a causa de los daños por sismos, las primeras reformas de las que hay constancia son las de 1740 , debidas al estallido de la Guerra del Asiento o de la Pragmática Sanción, momento en que se añadieron nuevas ta- 
pias (Gil Albarracín, 2014, p. 166). Sin embargo, no será hasta la segunda mitad del siglo XX cuando comiencen los trabajos de restauración y consolidación más o menos sistemáticos. Entre ellos, destacan los llevados a cabo por Francisco Prieto-Moreno Pardo, quien, a partir de 1961, llevó a cabo un proyecto de restauración financiado por el Ministerio de Educación Nacional en la muralla del Cerro de San Cristóbal y el tramo de La Hoya. En este momento, se reconstruyeron con tapial de hormigón los torreones 3 y 4 (desde el sur) del tramo de La Hoya y el lienzo que los une. En 1962, llevó a cabo la reconstrucción de los torreones 1 y 2 con tapial calicastrado, rematando la parte superior con ladrillos y la base con mampostería hormigonada. Dos años después, en 1964, se consolidó y restauró el sector oeste de la muralla (Romero Gallardo, 2010, pp. 494-495). Los trabajos de reparación continuaron de la mano del arquitecto $\mathrm{R}$. Puig Álvarez en 1979, llevándose a cabo una obra de restauración promovida por el Ministerio de Cultura. En los últimos 20 años se han sucedido varias actuaciones de reconstrucción parcial de esta muralla: en octubre de 2003, el arquitecto J. A. García Valera, junto con el químico A. Odriozola Romillo y los ingenieros P. Arozamena Cagigal y C. Moreno Galdó, realizaron un estudio de la estabilidad estructural de los muros y forjados, encargado por la Delegación de Cultura de Almería. En él se determinaron sus características estructurales para su musealización, analizando los esfuerzos mecánicos estáticos o dinámicos, así como su estabilidad. Se hizo un estudio patológico y se repararon los daños ocasionados por las distintas patologías, así como tres calicatas para conocer la cimentación de la muralla. Este estudio supuso el paso previo para la posterior restauración del conjunto. Al año siguiente, en 2004, el arquitecto J. Basterra Pinilla presentó un anteproyecto de intervención, donde se hacía un diagnóstico de la situación de la muralla de La Hoya (Basterra Pinilla, 2004). El proyecto se materializó en 2006, promovido por la Consejería de Cultura de la Junta de Andalucía. En él se procedió a la limpieza de los muros, con la eliminación de la vegetación, se sanearon y consolidaron los lienzos de muralla y torres, se repuso el material (mampostería y tapial) en los puntos en que fue necesario siguiendo las técnicas tradicionales -con mortero bastardo coloreado-, se consolidó el remate de la parte alta de los muros, se colocó la carpintería en los cierres y se restituyeron los forjados de las torres y cubiertas (Basterra Pinilla, 2006). El proyecto inicial fue modificado en 2008, simplificando las pasarelas y escaleras previstas, y colocando estructuras de metal fijas (de acero corten) que facilitaran su desmontaje. Asimismo, se repararon los daños estructurales del adarve, provocados por una gran grieta, y de diversas torres, adaptando sus cubiertas a los desaguies existentes. Por otro lado, se eliminaron elementos añadidos a la construcción, se dejaron vistos sus antiguos sistemas de drenaje y se modificó el sistema que se había previsto para drenar las aguas y evacuarlas hacia la vaguada (Basterra Pinilla, 2008). El empleo del acero corten se justificó desde el concepto de reversibilidad, legibilidad, y de aportación contemporánea (Cachorro Fernández, 2011, p. 13), aunque su uso ha ocasionado problemas de oxidación que han dado lugar a la presencia de manchas en varias torres y otros puntos de la muralla.

Debido a las recientes y continuas restauraciones llevadas a cabo en la muralla de La Hoya recogidas en este trabajo, puede concluirse que, en general, este bien se encuentra en buen estado de conservación, limitándose sus patologías sobre todo a las manchas producidas por el óxido del acero corten en algunas de sus torres. Esta situación se acentúa en las torres H-8 y H-10, sobre todo en su frente sureste y en algunos de sus paramentos adyacentes. Por ello, se recomienda como acción prioritaria de conservación la aplicación de un tratamiento de limpieza de las manchas de óxido. Como medidas de prevención, se recomienda la impermeabilización, protección y creación de sistemas de evacuación del agua de lluvia para proteger los paramentos de la humedad, así como el drenaje de la base de los muros para reducir las humedades por capilaridad. Como medidas de restauración, se recomienda la limpieza profunda para el eliminado de la suciedad adherida, los depósitos de polvo y las pinturas y grafitis, presentes en algunos puntos (aunque estos son más abundantes en el tramo de la muralla del Cerro de San Cristóbal, de- 
bido a la menor accesibilidad de la muralla de La Hoya), así como un estudio arqueológico del entorno y su adecuación, puesto que la zona se encuentra bastante degradada.

\section{Conclusiones}

La metodología seguida en el Proyecto PREFORTI se basa en un estudio integral y multidisciplinar de las fortificaciones de tierra, en aras de asegurar un mejor conocimiento de su historia, sus características constructivas, los riesgos naturales y antrópicos por los que se han visto afectadas, las diversas actuaciones llevadas a cabo en ellas y un análisis de su estado de conservación, a partir del cual realizar una serie de propuestas de conservación e intervención, incidiendo en las de carácter preventivo. Tras el análisis aquí recogido, podemos concluir que, en el caso de Almería, los daños ocasionados por sismos son los que más han comprometido el estado de conservación de su patrimonio militar de tierra. En este contexto, las cartografías de riesgo elaboradas en el marco del Proyecto PREFORTI, trabajando a una escala de microzonificación, vinculan los bienes analizados a su ámbito territorial, permitiendo un conocimiento más profundo de los diferentes riesgos que afectan al patrimonio de tierra. Las principales acciones a considerar teniendo en cuenta que la fórmula del riesgo es $\mathrm{R}$ (riesgo) $=\mathrm{V}$ (vulnerabilidad) $\mathrm{x} P$ (peligrosidad), y que no puede ser minimizada la acción de los factores de peligrosidad, significa que las acciones deben recaer en la reducción de la vulnerabilidad. De ahí que los esfuerzos deban dirigirse, entre otros, a la toma de datos de carácter mecánico del material, de la estructura (peso, inercia, periodo de vibración) y datos de vibración del suelo. De este modo, conocer la frecuencia de la estructura y del suelo posibilita adoptar medidas para que ambas sean lo más separadas posible en valores, con el fin de buscar el desacople en la frecuencia y por tanto evitar la resonancia. El análisis de tales parámetros condiciona el modelo de intervención ya que ésta, además de no dañar los valores patrimoniales de la obra, debe evitar el aumento de la vulnerabilidad adscrita a un aumento de la masa, de la altura del elemento o modificaciones en el periodo fundamental. En este contexto, los materiales aportados desde el Proyecto PREFORTI se convierten en una importante estrategia a seguir de cara a la conservación preventiva que garantice la preservación de este patrimonio.

\section{Agradecimientos}

Este estudio forma parte del proyecto $\mathrm{I}+\mathrm{D}+\mathrm{i}$ PREFORTI (BIA2015 69938-R) titulado "Metodología sostenible de conservación y mantenimiento de fortificaciones medievales de tierra del sudeste de la Península Ibérica. Diagnóstico y prevención ante riesgos naturales y antrópicos" financiado por la Agencia Estatal de Investigación (AEI) y el Fondo Europeo de Desarrollo Regional (FEDER).

\section{Notas}

${ }^{1}$ Entre los terremotos acaecidos en la época medieval previos al de 1487, destacan el seísmo de 1048-1049 relatado por al- 'Uḍīi, que afectó a la región de Vera y Los Vélez (Espinar Moreno, 1994, p. 129), así como el de 1406 en la misma región (Espinar Moreno, 1994, p. 131).

\section{Bibliography}

Alcocer Martínez, M. (1941). "Castillos y fortalezas del antiguo Reino de Granada", in Serie 3 $3^{a}$. Utilización de archivos españoles, $n^{\circ}$ 6, Publicaciones del Instituto General Franco para la Investigación Hispano-Árabe Ed., Madrid.

Basterra Pinilla, J. (2004). Anteproyecto para restauración de la Muralla de la Hoya - Almería, Expediente B043185 HP04BC, Dirección General de Bienes Culturales y Museos, Sevilla.

Basterra Pinilla, J. (2006). Proyecto básico y de ejecución Restauración de la Muralla de la Hoya, Almería, Expediente B043185 HP04BC, Delegación de Cultura de Almería, Almería.

Basterra Pinilla, J. (2008). Proyecto básico y de ejecución modificado. Restauración de la Muralla de la Hoya - Almería, Expediente B043185 HP04BC, Delegación de Cultura de Almería, Almería. 
Cachorro Fernández, E. (2011). "Intervención en la muralla de la Hoya”, Revista PH, 79, pp. 10-13.

Cara Barrionuevo, L.; Rodríguez López, J.Ma. (1992). "Prospección arqueológica superficial de la Alta Alpujarra almeriense y del Campo de Dalías oriental (Almería)”, in Anuario Arqueológico de Andalucía 1990, Consejería de Cultura / Junta de Andalucía Ed., Sevilla, vol. II, pp. 140-147.

Espinar Moreno, M. (1994). "Los estudios de sismicidad histórica en Andalucía: los terremotos históricos de la provincia de Almería”, in Posadas Chinchilla, A.M.; Vidal Sánchez, F., coords., El estudio de los terremotos en Almería, Estudios Almerienses Ed., Almería, pp. 115-180.

Espinosa Spínola, G.; Nicolás Martínez, Ma.M.; Torres Fernández, R.; Ureña Uceda, A. (2006). Guía artística de Almería y su provincia, Fundación José Manuel Lara-Diputación Eds., Almería.

García Asensio, E. (1908). Historia de la villa de Huércal-Overa y su comarca, Instituto de Estudios Hurcalenses Ed., Huércal-Overa, vol. II.

Gil Albarracín, A. (2014). Almería: la plaza de armas. Más de mil años de fortificaciones, Griselda Bonet Girabet Ed., Barcelona, pp. 151-304.

IAPH. (s.d.). Base de Datos del Patrimonio Inmueble de Andalucía. IAPH-Consejería de Cultura de la Junta de Andalucía Eds., Sevilla, in http://www.iaph.es/patrimonio-inmueble-andalucia/ (14 July 2017).

López Guzmán, R., coord. (2002). Arquitectura de al-Andalus (Almería, Granada, Jaén, Málaga), El Legado Andalusí-Comares Eds., Granada.

Marcos Cobaleda, M.; Gutiérrez Carrillo, Ma.L. (2019). Inventario Fortificaciones Medievales de Tierra. Almería. Proyecto PREFORTI.

Marcos Cobaleda, M.; Gutiérrez Carrillo, Ma.L. (en revisión). "Military Architecture in Southeast Al-Andalus during the First Half of the $12^{\text {th }}$ Century: Analysis of Their Remains and Risks of Conservation", International Journal of Architectural Heritage (IJAH).

Martínez Solares, J.M. (2001). Los efectos en España del terremoto de Lisboa (1 de noviembre de 1755), Dirección General del Instituto Geográfico Nacional-Ministerio de Fomento Eds., Madrid.

Romero Gallardo, A. (2010). La restauración arquitectónica en el periodo franquista: la figura de Francisco PrietoMoreno y Pardo, Tesis Doctoral, Universidad de Granada, Granada.

Silva, P.G.; Rodríguez Pascua M.A., eds. (2014). Catálogo de los efectos geológicos de los terremotos en España, Instituto Geológico y Minero de España (IGME)-Asociación Española para el Estudio del Cuaternario (AEQUA) Eds., Madrid.

Suárez Márquez, Á. coord. (2009). Monografías del Conjunto Monumental de la Alcazaba II. Construir en alAndalus, Consejería de Cultura de la Junta de Andalucía Ed., Sevilla.

Torres López, R. de. (1992). Restauración de la Alcazaba de Almería. Quinta fase de intervenciones, Dirección General de Bienes Culturales y Museos Ed., Sevilla. 